\title{
Nephrogenic adenoma in elderly patients: Three case reports
}

\author{
TORU SAKATANI ${ }^{1 *}$, YASUSHI ADACHI ${ }^{2,3 *}$, NORIKO SAKAIDA ${ }^{4,5}$, TAKESHI ATSUTA $^{6}$, \\ TOSHIHIRO MAGARIBUCHI ${ }^{1}$, YOJI TAKI ${ }^{1}$, YORIKA NAKANO ${ }^{7},{\text { MING } \text { LI }^{3} \text { and SUSUMU IKEHARA }}^{3}$
}

\author{
Departments of ${ }^{1}$ Urology and ${ }^{2}$ Surgical Pathology, Toyooka Hospital, Toyooka, Hyogo 668-8501; \\ ${ }^{3}$ Department of Stem Cell Disorders, Kansai Medical University, Hirakata, Osaka 573-1010; ${ }^{4}$ Department \\ of Diagnostic Pathology, Kakogawa West City Hospital, Kakogawa, Hyogo 675-8611; ${ }^{5}$ Department of Diagnostic Pathology, \\ Osaka City University Graduate School of Medicine, Osaka 565-0871; ${ }^{6}$ Department of Urology, \\ Kurashiki Central Hospital, Kurashiki, Okayama 710-8602; ${ }^{7}$ Department of Surgical Pathology, \\ Kansai Medical University, Hirakata Hospital, Hirakata, Osaka 573-1191, Japan
}

Received February 23, 2016; Accepted April 18, 2016

DOI: $10.3892 / \mathrm{mco} .2016 .930$

\begin{abstract}
Nephrogenic adenoma (NA), referred to as nephrogenic metaplasia, is a rare benign lesion of the urinary tract. NA is histologically characterized by tubular and papillary formations lined by low cuboidal to columnar epithelial cells. NA is also immunohistochemically characterized by positivity for paired box (PAX) 2, PAX8 and cytokeratin 7, and negative for P63 and prostate-specific antigen. In this study, we present 3 cases of NA arising in the urinary bladder of elderly male patients with predisposing factors: Patient 1 had undergone transurethral lithotripsy due to a ureteral stone; patient 2 had undergone transurethral resection of a urothelial carcinoma in the urinary bladder; and patient 3 had been treated with Bacillus-Calmettle-Guérin due to a urothelial carcinoma in the urinary bladder. The characteristics of the NAs of our 3 cases were histologically and immunohistologically consistent with previously reported cases, although 1 patient exhibited a pseudoinvasive pattern. Since NA is a tumor-like benign lesion, it should be clearly differentiated morphologically and immunohistologically from other tumors arising in the urinary tract and from invasion by prostate cancer.
\end{abstract}

\section{Introduction}

Nephrogenic adenoma (NA) is an uncommon benign lesion of the urothelial mucosa of the urinary tract $(1,2)$. In 1949, Davis reported the first identified case of NA as a "hamartoma of the urinary bladder' (3). In 1950, Fiedman and Kuhlenbeck

Correspondence to: Professor Susumu Ikehara, Department of Stem Cell Disorders, Kansai Medical University, 2-5-1 Shinmachi, Hirakata, Osaka 573-1010, Japan

E-mail: ikehara@hirakata.kmu.ac.jp

${ }^{*}$ Contributed equally

Key words: nephrogenic adenoma, urinary bladder, elderly patients, predisposing factors, immunohistochemistry named this lesion 'nephrogenic adenoma' based on its histological similarity to renal tubules (4). It has been reported that NA mainly arises in the urinary bladder $(68.6 \%)$ and urethra (13.3\%), but also in the ureter (8.2\%), renal pelvis $(8.2 \%)$ and, rarely, in the prostate (2\%) (5). NAs exhibit a male predominance, with a male:female ratio of $\sim 3.6: 1$. When NA occurs in the female urethra, approximately one-fourth of the cases are associated with a urethral diverticulum $(6,7)$.

From a study including 134 cases of NA, Lopez reported that the mean age of NA patients was 66 years, with a range of 14-96 years (5). Furthermore, Husain et al reported 18 cases of NA in pediatric patients, ranging in age from 2 to 19 years (8), whereas Kao et al reported 21 cases of pediatric NAs, with an age range of 2-16 years (9). The usual clinical presentation is hematuria, dysuria and frequency of micturition (10). Several predisposing factors have been reported to be associated with NA, in adults as well as in children, including genitourinary trauma, chronic inflammation, prior surgery, renal calculi, repeated instrumentation, irritated anatomical anomalies and pelvic irradiation, bacillus Calmette-Guérin (BCG) immunotherapy and diverticuli $(1,8,9,11,12)$.

We herein present 3 cases of NA arising in the urinary bladder of elderly male patients.

\section{Case reports}

Case 1. A 64-year-old man consulted Toyooka Hospital (Hyogo, Japan) due to gross hematuria. The patient had undergone transurethral lithotripsy (TUL) due to a left ureteral stone 6 years prior to the consultation, followed by transurethral resection of the prostate (TUR-P) for benign prostatic hyperplasia (BPH) 3 years prior. The patient underwent cystoscopy, which revealed a small papillary lesion in the bladder neck (Fig. 1A). The lesion was resected and histological examination of the resected lesion revealed papillary and tubular formations, lined by low cuboidal to flattened epithelial cells (Fig. 1B). The epithelial cells were immunohistologically positive for cytokeratin (CK) 7, paired box (PAX) 8 and P504S, and negative for P63 and prostate-specific antigen (PSA) (Fig. 1C and D and data not shown). Therefore, the lesion was diagnosed as NA. In the 3 years (to date) since 

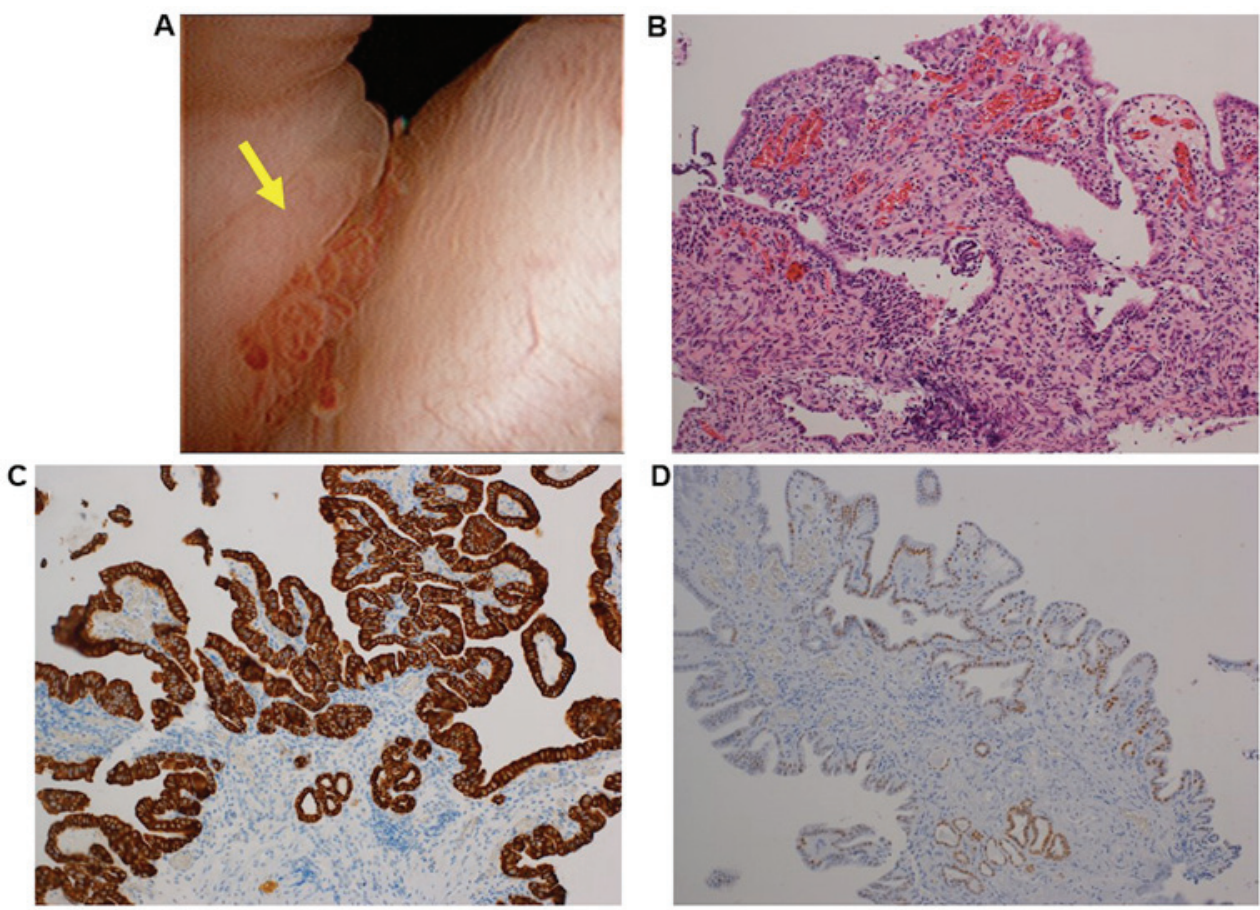

Figure 1. Macroscopic view by cystoscopy and histological examination of the lesion in case 1. (A) Macroscopic view by cystoscopy: A small papillary lesion was observed in the neck of the urinary bladder (arrow). Microscopic examination of the specimen obtained by transuretheral resection was also performed. (B) On hematoxylin and eosin-stained sections, papillary and tubular lesions were observed. Immunohistochemical examination revealed that the epithelial cells of the lesion stained positive for (C) cytokeratin 7 and (D) paired box 8 (magnification, x20).
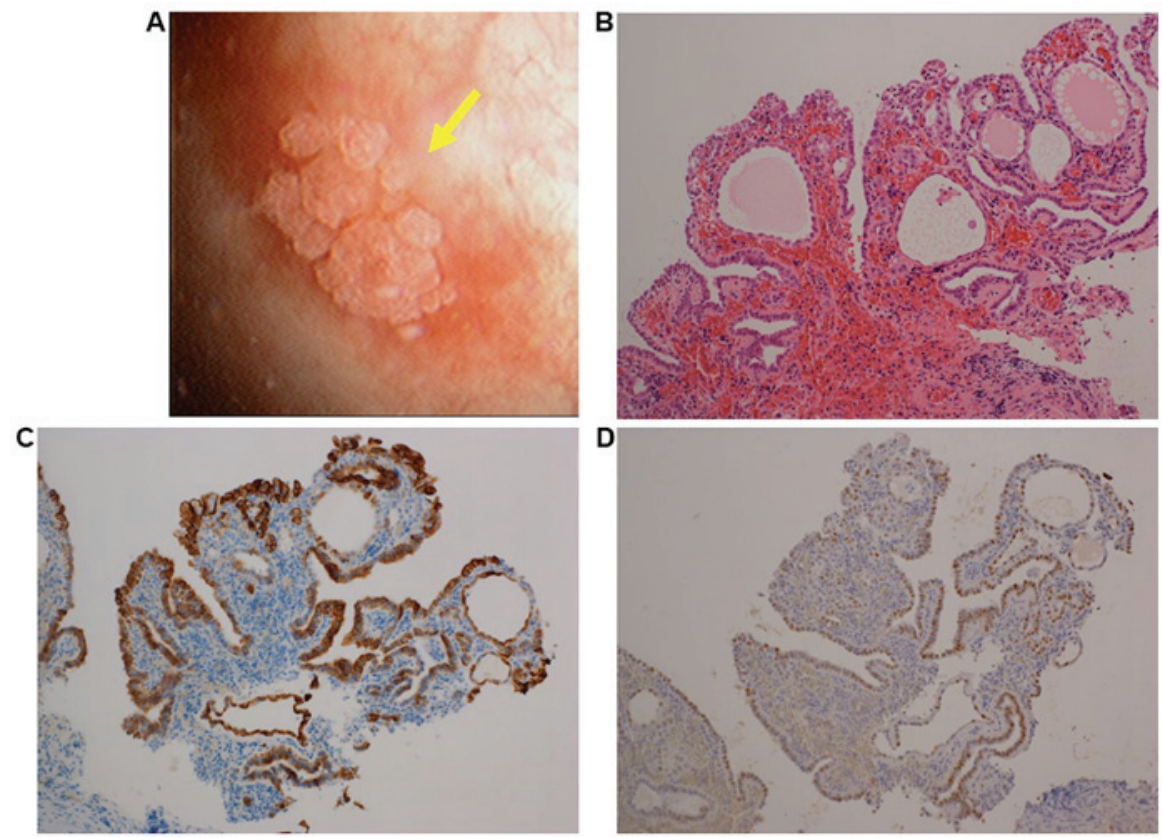

Figure 2. Macroscopic view by cystoscopy and histological examination of the lesion in case 2. (A) Macroscopical view by cystoscopy: A small papillary lesion was observed in the left lateral wall of the urinary bladder (arrow). Microscopic examination of the specimen obtained by transuretheral resection was also performed. (B) On hematoxylin and eosin-stained sections, papillary and tubular lesions were observed. Immunohistochemical examination revealed that the epithelial cells of the lesion stained positive for (C) cytokeratin 7 and (D) paired box 8 (magnification, x20).

the resection of the NA, the patient has shown no signs of NA recurrence.

Case 2. The urinary bladder of an 82-year-old man had been examined using a cystoscope at Toyooka Hospital during a follow-up visit for urothelial carcinoma of the left lateral wall of the urinary bladder, which had been treated with TUR 1 year prior. The patient had also been treated with peritoneal dialysis due to end-stage renal failure from approximately the same time as TUR onwards. A small sessile papillary lesion was identified in the left lateral wall of the urinary bladder (Fig. 2A). The lesion was transurethrally resected 

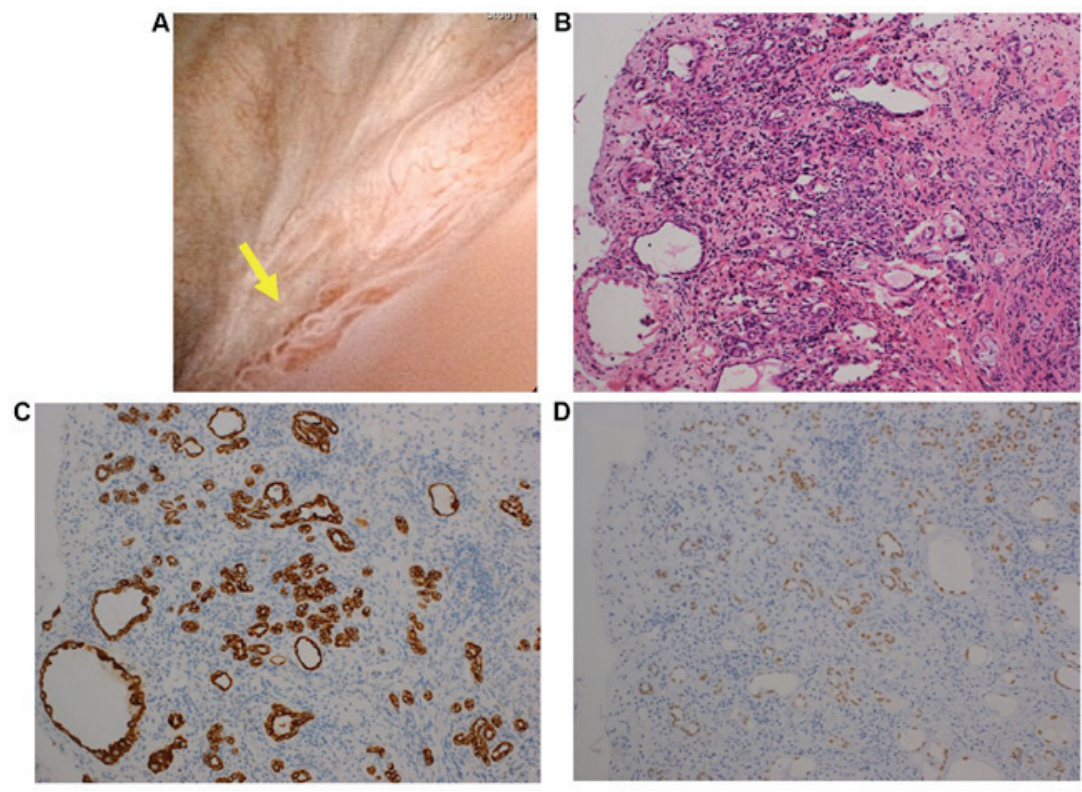

Figure 3. Macroscopic view by cystoscopy and histological examination of the lesion in case 3. (A) Macroscopic view by a cystoscopy: A small papillary lesion was observed in the left lateral wall of the urinary bladder (arrow). (B) Area of the lesion with tubular pattern (hematoxylin and eosin staining). The tubular structures are observed in the interstitial tissue of the urinary bladder. Immunohistochemical examination revealed that the epithelial cells of the tubular structures stained positive for (C) cytokeratin 7 and (D) paired box 8 (magnification, $\mathrm{x} 20$ ).

and histological examination revealed a lesion similar to that described in case 1, mainly composed of papillary and partially tubular structures lined by low cuboidal to flattened epithelial cells (Fig. 2B). The epithelial cells were immunohistologically positive for CK7, PAX8 and P504S, and negative for P63 and PSA (Fig. 2C and D and data not shown). Therefore, the lesion was diagnosed as NA.

Three years after the TUR for NA the patient succumbed to candida pneumonia that was unrelated to the NA or bladder cancer.

Case 3. An 87-year-old man underwent TUR of an urothelial carcinoma at the trigone of the urinary bladder, followed by intravesical chemotherapy with mitomycin $\mathrm{C}$ at Toyooka Hospital. Twelve months later, there was a recurrence of the urothelial carcinoma in the urinary bladder. TUR and intravesical therapy with BCG were performed. Six months after the recurrence, a papillary lesion was observed around the right ureteral orifice (Fig. 3A). The lesion was transurethrally resected. Histologically, part of the lesion exhibited a papillary pattern, similar to cases 1 and 2, whereas another part exhibited tubular structures in the interstitial tissue of the urinary bladder, histologically mimicking invasion by prostate cancer (Fig. 3B). However, immunohistochemical staining revealed that the epithelial cells comprising the tubular structure were positive for CK7 and PAX8, but not for P63 or PSA (Fig. 3C and D and data not shown), suggesting the lesion was also a NA.

In the 6 months (to date) since the resection of the NA, the patient has shown no signs of NA recurrence.

\section{Discussion}

López et al analyzed 134 cases of NA with a mean patient age of 66 years, suggesting that elderly individuals appear to be more prone to development of NA (5). However, the studies of Husain et al and Kao et al involved pediatric cases of NA $(8,9)$. Several predisposing factors have been reported to be associated with NA, in adults as well as in children $(1,8,9,11,12)$. Our patients had also undergone various treatments prior to the development of the NAs; 1 patient was subjected to TUL due to a ureteral stone and TUR-P due to BPH; 1 patient had received TUR due to urothelial carcinoma in the urinary bladder; and the third patient had received TUR due to urothelial carcinoma in the urinary bladder, followed by treatment with BCG. Regarding the mechanisms underlying the origin of NA, it has been reported that the NAs arising in female recipients of renal transplantations from male donors display a male chromosomal status on in situ hybridization, while NAs arising in the male recipients of renal transplantations from female donors display a female chromosomal status (13). However, the stromal components of NA exhibit the sex chromosome status of the recipients. These results suggest that epithelial cells in NA are derived from the renal tubules, such that the detached epithelial cells in the renal tubules are able to become engrafted in the injured urinary tract, from which the urothelial epithelium has disappeared.

It has been reported that the epithelial cells of NA are similar to those of renal tubules and that the epithelial cells of NA express PAX2, PAX8 and CK7, but not p63 or PSA (2). The expression pattern of antigens is similar to that of renal tubules. These results also support the theory of NAs originating from the renal tubules. All our patients also exhibited the same patterns of expression of these antigens.

It has also been reported that NA occasionally mimics prostatic adenocarcinoma, due to the pseudoinvasive pattern of small tubules lacking a basal cell layer $(14,15)$. Kunju presented an image of an NA in the superficial muscle layer of the urinary bladder (15). In our case 3, the NA was found in the interstitial tissue in the urinary bladder. Based on the theory 
that NA originates in the renal tubules, it is suggested that epithelial cells from the renal tubules become embedded in the urothelial tract wall followed by restoration of the structure of the wall. During the restoration process, the epithelial cells of the renal tubules may become implanted into the urothelial tract wall, even as deep as the muscle layer.

In the present study, we presented 3 cases of NA in elderly male patients with predisposing factors. Since NAs are benign lesions, it is important to make a definitive differential diagnosis of NAs based on their morphology, immunohistochemistry and the patient's history, particularly in cases with any predisposing factors.

The patients or their families provided informed consent for the publication of the case details, and the Ethics Committee of Toyooka Hospital approved the publication of this study.

\section{Acknowledgements}

We would like to thank Ms. K. Ando (Department of Stem Cell Disorders, Kansai Medical University) and Mr. Hilary Eastwick-Field for the preparation of this manuscript. We would also like to thank Ms. H. Ogaki, Mr. K. Nagaoka, Mr. T. Kuge, Mr. H. Takenaka and Ms. S. Kawasaki at Toyooka Hospital for their expert technical assistance.

\section{References}

1. Amin W and Parwani AV: Nephrogenic adenoma. Pathol Res Pract 206: 659-662, 2010 .

2. Alexiev BA and LeVea CM: Nephrogenic adenoma of the urinary tract: A review. Int J Surg Pathol 20: 123-131, 2012.

3. Davis TA: Hamartoma of the urinary bladder. Northwest Med 48: $182-185,1949$.

4. Friedman NB and Kuhlenbeck H: Adenomatoid tumors of the bladder reproducing renal structures (nephrogenic adenomas). J Urol 64: 657-670, 1950.
5. López JI, Schiavo-Lena M, Corominas-Cishek A, Yagüe A, Bauleth K, Guarch R, Hes O and Tardanico R: Nephrogenic adenoma of the urinary tract: Clinical, histological, and immunohistochemical characteristics. Virchows Arch 463: 819-825, 2013.

6. Ford TF, Watson GM and Cameron KM: Adenomatous metaplasia (nephrogenic adenoma) of urothelium. An analysis of 70 cases. Br J Urol 57: 427-433, 1985.

7. Oliva E and Young RH: Nephrogenic adenoma of the urinary tract: A review of the microscopic appearance of 80 cases with emphasis on unusual features. Mod Pathol 8: 722-730, 1995.

8. Husain AN, Armin AR and Schuster GA: Nephrogenic metaplasia of urinary tract in children: Report of three cases and review of the literature. Pediatr Pathol 8: 293-300, 1988.

9. Kao CS, Kum JB, Fan R, Grignon DJ, Eble JN and Idrees MT: Nephrogenic adenomas in pediatric patients: A morphologic and immunohistochemical study of 21 cases. Pediatr Dev Pathol 16: 80-85, 2013.

10. Xiao GQ, Burstein DE, Miller LK and Unger PD: Nephrogenic adenoma: Immunohistochemical evaluation for its etiology and differentiation from prostatic adenocarcinoma. Arch Pathol Lab Med 130: 805-810, 2006.

11. Stilmant MM and Siroky MB: Nephrogenic adenoma associated with intravesical bacillus Calmette-Guerin treatment: A report of 2 cases. J Urol 135: 359-361, 1986.

12. Gujral H, Chen H and Ferzandi TR: Nephrogenic adenoma in a urethral diverticulum. Female Pelvic Med Reconstr Surg 20: e12-e14, 2014.

13. Mazal PR, Schaufler R, Altenhuber-Müller R, Haitel A, Watschinger B, Kratzik C, Krupitza G, Regele H, Meisl FT, Zechner O, et al: Derivation of nephrogenic adenomas from renal tubular cells in kidney-transplant recipients. N Engl J Med 347: 653-659, 2002.

14. Skinnider BF, Oliva E, Young RH and Amin MB: Expression of alpha-methylacyl-CoA racemase (P504S) in nephrogenic adenoma: A significant immunohistochemical pitfall compounding the differential diagnosis with prostatic adenocarcinoma. Am J Surg Pathol 28: 701-705, 2004.

15. Kunju LP: Nephrogenic adenoma: Report of a case and review of morphologic mimics. Arch Pathol Lab Med 134: 1455-1459, 2010. 